\title{
The pH-static Enzyme Sensor: Design of the pH Control System
}

\author{
BART H. VAN DER SCHOOT, HANS VOORTHUYZEN and PIET BERGVELD \\ Faculty of Electrical Engineering, University of Twente, P.O. Box 217, NL-7500 AE Enschede (The Netherlands)
}

\begin{abstract}
The pH-static enzyme sensor offers a solution to the buffer dependency of ISFET-based enzyme sensors. A continuous coulometric titration of the reaction products keeps the $\mathrm{pH}$ in the enzymatic membrane at a constant level. This paper presents an automatic system to control the compensating current that is a direct measure for the substrate concentration.
\end{abstract}

\section{Introduction}

The response of enzyme sensors based on the measurement of a $\mathrm{pH}$ change in an enzymatic membrane depends on the buffer capacity and the initial $\mathrm{pH}$ of the sample solution. For a given substrate concentration, the response of a sensor is determined by the buffer concentration. Moreover, as the enzyme kinetics are $\mathrm{pH}$ dependent, the response is nonlinear and the useful concentration range is limited. In a previous paper [1] a possible solution to this problem was presented. The pH-static urea sensor continuously neutralizes the alkaline products of the enzyme reaction through the generation of protons at a noblemetal electrode incorporated in the enzymatic membrane. The $\mathrm{pH}$ inside the membrane is thus controlled at a constant level, this process being monitored with an underlying $\mathrm{pH}$-sensitive ISFET. The relation between the urea concentration and the proton-generating current is linear, the response is buffer independent and the linear range is expanded.

The dynamic response of the $\mathrm{pH}$-static enzyme sensor depends on the optimal performance of the control system used, in connection with the sensor-actuator design. For the preliminary results, as presented in ref. 1 , the $\mathrm{pH}$ was controlled by manual adjustment of the current source. In this paper an automatic control system for the sensor is presented.

\section{Practical Set-up of the Sensor}

The pH-static enzyme sensor is based on a chemical sensor-actuator system consisting of an ISFET on which a thin-film platinum electrode is deposited that closely surrounds the $\mathrm{pH}$-sensitive gate area of the transistor [2]. On top of this system a membrane of polyacrylamide (PAA) with a thickness of $200 \mu \mathrm{m}$ is applied in which the enzyme urease is entrapped [3]. Figure 1 shows a cross section of the sensor.

When urea is added to the solution, the $\mathrm{pH}$ in the membrane will increase according to

$$
\text { urea }+3 \mathrm{H}_{2} \mathrm{O} \stackrel{\text { urease }}{\longrightarrow} 2 \mathrm{NH}_{4}^{+}+\mathrm{HCO}_{3}^{-}+\mathrm{OH}^{-}
$$

The magnitude of the increase in $\mathrm{pH}$ is strongly dependent on the buffer capacity of the solution. To overcome this problem, the $\mathrm{pH}$-static enzyme sensor performs a continuous titration of the hydroxyl ions produced in reaction (1). $\mathrm{H}^{+}$ions are produced at the platinum electrode by the electrolysis of water through a controlled current source. The rate of $\mathrm{H}^{+}$production is governed by a digital control system that compares the $\mathrm{pH}$ inside the membrane to that of the sample solution as measured by a reference ISFET. Figure 2 shows a diagram of the total system.

\section{Design of the Control System}

For an optimal design of the controller, the transfer function between the actuator current and the membrane $\mathrm{pH}$ has to be known. Similar to the 'classical' ISFET-based enzyme sensor, the magnitude of the $\mathrm{pH}$ change for a certain actuator current is determined by the buffer capacity $\beta$ of the solution. In Fig. 3(a) the response on a stepwise change in actuator current is shown for various concentrations of phosphate buffer, $\mathrm{pH}=7$. Figure $3(\mathrm{~b})$ shows a similar experiment for a number of values for the actuator current at

(C) Elsevier Sequoia/Printed in The Netherlands 


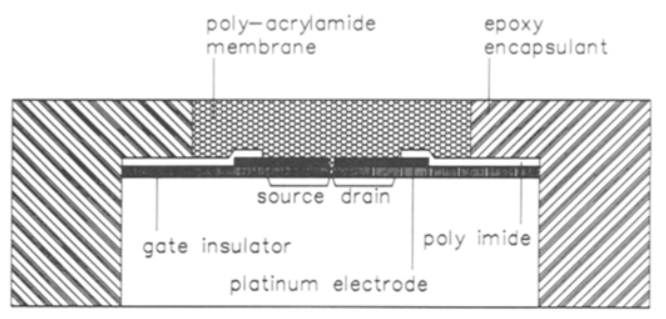

Fig. 1. Cross section of the pH-static enzyme sensor.

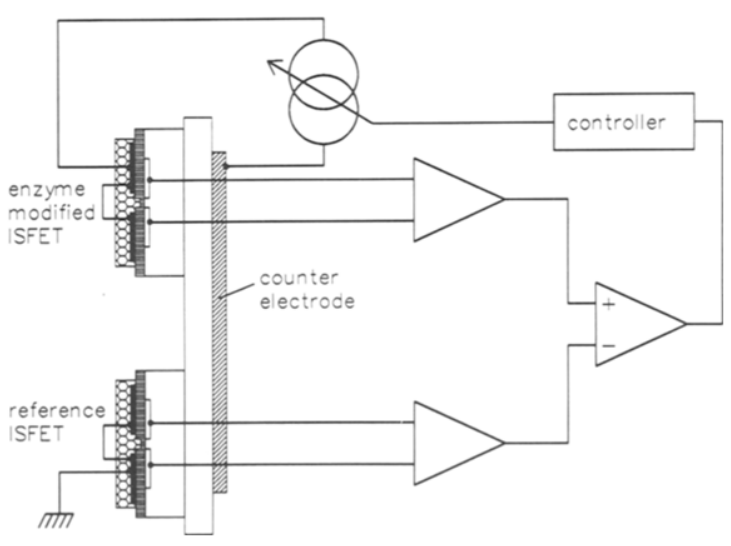

Fig. 2. Experimental set-up to control the $\mathrm{pH}$ in the membrane of the sensor.

a fixed buffer concentration. From these measurements both the static and the dynamic behaviour of the system can be determined.

Considering the equilibrium state that is reached after a change in actuator current, it can be seen that for small $\mathrm{pH}$ changes this change is linearly related to the current $I$ and inversely proportional to the buffer capacity $\beta$.

As can be seen from Fig. 1, a certain distance exists between the $\mathrm{pH}$ sensing gate of the ISFET and the actuator electrode. This implies that there will be a delay, $T_{\mathrm{d}}$, before the generated ions are diffused to the gate and can be measured. If the time scale of Figs. 3(a) and 3(b) is expanded around $t=0$, the value of $T_{\mathrm{d}}$ can be estimated. After this initial delay, the change in $\mathrm{pH}$ can be approximated as a first-order process with a time constant $\tau$. Thus the response for $t>T_{\mathrm{d}}$ on a step-wise applied actuator current $I$ at time $t=0$ can be written as

$$
\mathrm{pH}_{t}=\mathrm{pH}_{0}+A \frac{1}{\beta}\left[1-\exp \left(-\left(t-T_{\mathrm{d}}\right) / \tau\right)\right] I
$$

where $A$ is a constant ( $\mathrm{moll}^{-1} \mathrm{~A}^{-1}$ ) that is determined by the active area of the actuator electrode, the thickness of the membrane and the diffusion constants of the buffering components in the membrane.

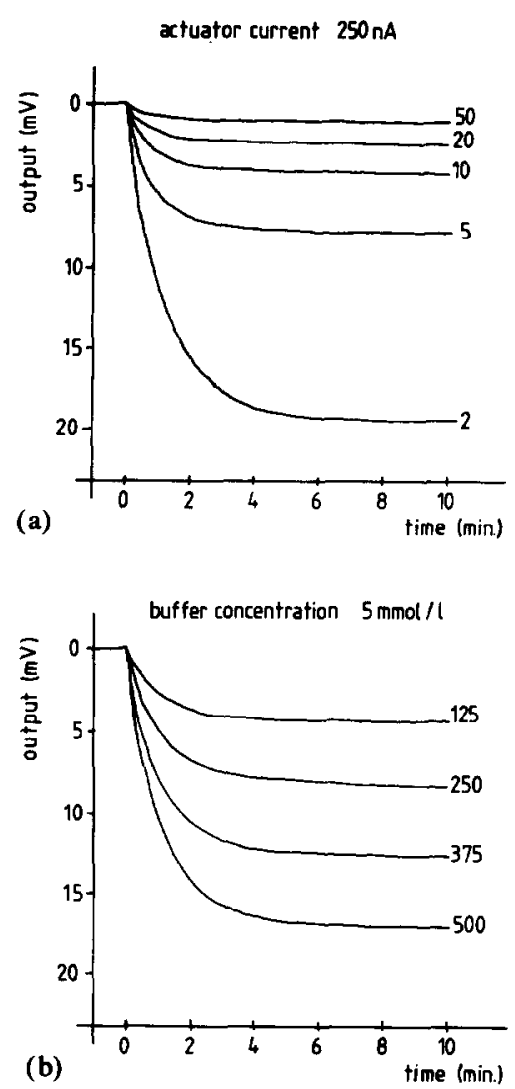

Fig. 3. (a) $\mathrm{pH}$ change in the membrane after a step-wise change in generating current for various concentrations of a phosphate buffer ( $\mathrm{pH}=7$ ). Buffer concentrations are given in mmol I-l (b) $\mathrm{pH}$ change in the membrane for various values of the generating current. Generating current is given in $\mathbf{n A}$.

The output voltage of the ISFET amplifier is measured with an a.c. to d.c. converter with a sampling interval $T_{\mathrm{s}}$. It can be shown that for low frequencies as compared to $1 / 2 \pi T_{\mathrm{s}}$ this may be considered as the introduction of an additional time delay of $T_{\mathrm{s}} / 2 \mathrm{~s}$.

In the frequency domain, the transfer function $H_{\mathrm{a}}$ between the actuator current and the ISFET output voltage can be written as

$H_{\mathrm{a}}(j \omega)=\frac{V_{\text {out }}(j \omega)}{I(j \omega)}=A \frac{\exp \left(-j \omega\left(T_{\mathrm{d}}+T_{\mathrm{s}} / 2\right)\right)}{1+j \omega \tau} \frac{B}{\beta}$

where $j \omega$ is the Laplace operator and $B$ is the sensitivity of the ISFET in connection with its amplifier $\left(\mathrm{mV} \mathrm{pH}^{-1}\right)$.

Now that the transfer function $H_{\mathrm{a}}$ is known, a controller with transfer function $H_{c}$ can be added to the system so that the $\mathrm{pH}$ in the enzyme membrane can be kept at a constant value. From eqn. (3) it can be seen that there are two elements that have to be taken into account for the design 
of the controller. The influence of the time constant $\tau$, which occurs in the transfer function as a factor $(1+j \omega \tau)^{-1}$, can be compensated by a differentiating element $(1+j \omega \tau)$ in the controller.

The second factor that has to be compensated is the delay time $\left(T_{\mathrm{d}}+T_{\mathrm{s}} / 2\right)$. In order to minimize the static error and to obtain a stable control, an integrating element $K / j \omega \tau_{\mathrm{i}}$ is added. The complete transfer function of the controller is now given by:

$H_{\mathrm{c}}(j \omega)=K \frac{(1+j \omega \tau)}{j \omega \tau_{\mathrm{i}}}$

in which $\tau_{\mathrm{i}}$ is the integration time and $K$ is the amplification of the controller.

In Fig. 4 a schematic representation of the control system is given. In this Figure, $V_{\text {set }}$ is the desired output voltage of the ISFET amplifier, $\epsilon$ is the error signal, $I$ is the control current, $C$ is the concentration of the produced $\mathrm{H}^{+}$or $\mathrm{OH}^{-}$ions and $V_{\text {out }}$ is the true value of the output voltage that represents the actual membrane $\mathrm{pH}$.

The open-loop amplification $G(j \omega)$ of the system is given by

$$
\begin{aligned}
G(j \omega) & =\frac{V_{\text {out }}(j \omega)}{\epsilon(j \omega)}=H_{\mathrm{c}} H_{\mathrm{a}} \\
& =K A \frac{\exp \left(-j \omega\left(T_{\mathrm{d}}+T_{\mathrm{s}} / 2\right)\right)}{j \omega \tau_{\mathrm{i}}} \frac{B}{\beta}
\end{aligned}
$$

and the closed-loop amplification $\dot{H}(j \omega)$ is given by

$\stackrel{\circ}{H}(j \omega)=\frac{V_{\text {out }}(j \omega)}{V_{\text {set }}(j \omega)}=\frac{G(j \omega)}{1+G(j \omega)}$

The control is optimal if $\stackrel{H}{H}(j \omega)$ approaches unity and thus $G(j \omega)$ has to be maximized to the general control criteria for stable closed-loop systems. The maximal amplification is determined by the phase shift at the circular frequency $\omega$ where the modulus of $G(j \omega)$ equals 1 . For a stable control, this phase shift should be smaller than $-180^{\circ}$ and it is common practice to choose a phase margin of $45^{\circ}$ to $60^{\circ}$. The phase shift of $G(j \omega)$ is given by

$\Phi(j \omega)=-\frac{\pi}{2}-\left[\omega\left(T_{\mathrm{d}}+T_{\mathrm{s}} / 2\right)\right]$

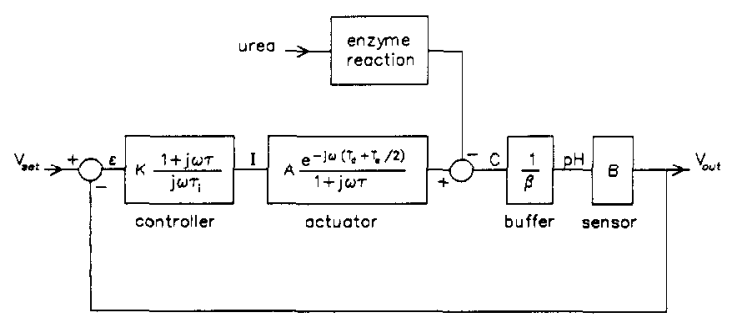

Fig. 4. The complete control loop for the pH-static enzyme sensor.
For a phase margin of $60^{\circ}$ or $\Phi(j \omega)=-2 / 3 \pi, \omega$ is given by

$$
\omega=\frac{\pi}{6\left(T_{\mathrm{d}}+T_{\mathrm{s}} / 2\right)}
$$

The modulus of $G(j \omega)$ is given by

$|G(j \omega)|=K A \frac{B}{\beta} \frac{1}{\omega \tau_{\mathrm{i}}}$

At the value of $\omega$ given in eqn. (8), $|G(j \omega)|$ should be equal to 1 and thus the maximal amplification $K$ can be calculated according to

$$
K=\frac{\pi \tau_{\mathrm{i}}}{6\left(T_{\mathrm{d}}+T_{\mathrm{s}} / 2\right)} \frac{\beta}{A B}
$$

The integration time $\tau_{\mathrm{i}}$ can be freely chosen and the values for $A$ and $T_{\mathrm{d}}$ can be determined from the experiment illustrated in Fig. 3(a) and (b).

\section{Realization of the controller}

The digital controller consists of an Apple IIe microcomputer that measures the output of the differential amplifier, as shown in Fig. 2, through an A/D converter. The current source has to be kept floating with respect to the $\mathrm{pH}$-measuring circuit. Therefore the current source is battery powered and connected to the controller via optocouplers. The output current is modulated by the duty cycle at which the constant current source is switched on and off. A comparable system has been used before to control the $\mathrm{pH}$ in $50 \mathrm{ml}$ of unbuffered solution [4].

Equation (4) gives the transfer function $H_{\mathrm{c}}=I(j \omega) / \epsilon(j \omega)$ of the controller in the frequency domain which can also be written as

$j \omega I(j \omega)=\frac{K}{\tau_{\mathrm{j}}}(1+j \omega \tau) \epsilon(j \omega)$

After inverse Fourier transformation, this yields in the time domain

$\frac{\mathrm{d} I(t)}{\mathrm{d} t}=\frac{K}{\tau_{\mathrm{i}}}\left(\epsilon(t)+\tau \frac{\mathrm{d} \epsilon(t)}{\mathrm{d} t}\right)$

Written in discrete form, this results in the control algorithm that calculates the new setting $I(t)$ for the current source after each sample interval $T_{\mathrm{s}}$

$$
I(t)=I\left(t-T_{\mathrm{s}}\right)+\frac{K T_{\mathrm{s}}}{\tau_{\mathrm{i}}}\left(\epsilon(t)+\tau \frac{\left(\epsilon(t)-\epsilon\left(t-T_{\mathrm{s}}\right)\right.}{T_{\mathrm{s}}}\right)
$$

The control program is written in Applesoft BASIC in connection with some machine language routines for input/output and timing. The output voltage $V_{\text {out }}$ and the control current $I$ are made available as analog signals via $D / A$ converters. 
TABLE 1. Essential parameters of the system

\begin{tabular}{llll}
\hline Parameter & & Value & Units \\
\hline$A$ & constant (see eqn. (2)) & $1.59 \times 10^{3}$ & $\mathrm{~mol} \mathrm{l}^{-1} \mathrm{~A}^{-1}$ \\
$B$ & ISFET sensitivity & 55 & $\mathrm{mV} \mathrm{pH}^{-1}$ \\
$T_{\mathrm{s}}$ & sample interval & 0.5 & $\mathrm{~s}$ \\
$T_{\mathrm{d}}$ & delay time & 2 & $\mathrm{~s}$ \\
$\tau$ & open-loop time constant & 60 & $\mathrm{~s}$ \\
$\tau_{\mathrm{i}}$ & integration time & $10^{6}$ & $\mathrm{~s}$ \\
$K$ & controller amplification & $2.66 \times \beta$ & $\mu \mathrm{A} \mathrm{mV}-1$ \\
\hline
\end{tabular}

As can be seen from eqn. (10), the maximum amplification $K$ depends on the buffer capacity of the solution. Therefore, to obtain an optimal value for $K, \beta$ has either to be known or should be determined. Because the system is well characterized according to eqn. (2), $\beta$ can be easily derived from the response on a short current pulse superimposed on the actual control current. Thus, while the system starts its controlling action with a low value of $K$, it is able to adapt the amplification to the actual sample solution.

\section{Results and Discussion}

In Table 1 the essential parameters of the system are summarized. The values of $A, T_{\mathrm{d}}$ and $\tau$ are derived from the experiments illustrated in Figs. 3 (a) and 3(b), B depends on the type of ISFET used and $T_{\mathrm{s}}$ and $\tau_{\mathrm{i}}$ are determined by the control program. When these parameters are known, the value of $K$ can be calculated according to eqn. (10).

Figure 5 demonstrates the closed-loop behaviour of the system. Curve A shows the control current and curve $B$ gives the output voltage of the ISFET. At the moments indicated by the arrows, the desired output voltage $V_{\text {set }}$ is changed from 0 to $8 \mathrm{mV}$ and back to $0 \mathrm{mV}$ respectively. It can be seen that the newly desired voltage is reached within $20 \mathrm{~s}$.

Figure 6 shows the pH-static enzyme sensor in operation. Again, curve A represents the control current and B is the ISFET output voltage. Starting with a low value for $K$, the system first

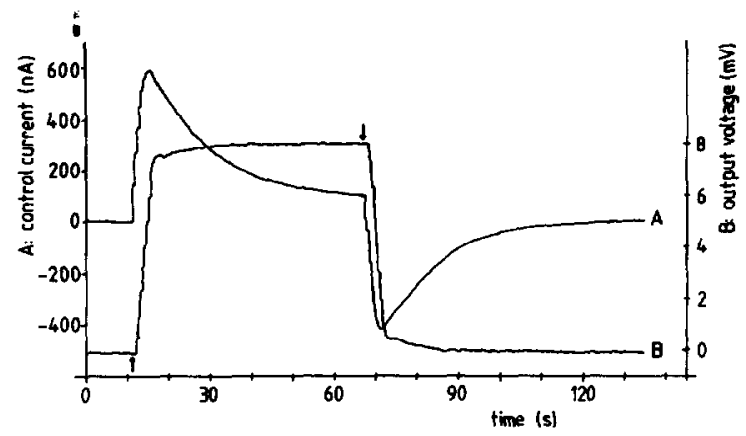

Fig. 5. Closed-loop behaviour of the system.

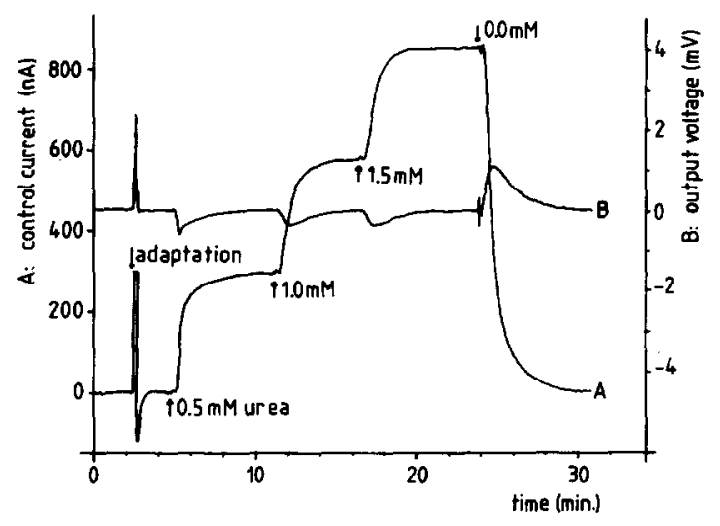

Fig. 6. The response of the sensor to step-wise changes in urea concentration.

determines the optimal value of the controller amplification from a superimposed current pulse of $10 \mathrm{~s}$. After that, urea is injected in the solution at the moments indicated by the arrows and finally the sensor is placed in a fresh solution that contains no urea. It can be seen that the response to a step-wise change in the urea concentration is complete within $3 \mathrm{~min}$. During the control action, the output voltage remains constant within approximately $1 \mathrm{mV}$ or $0.02 \mathrm{pH}$ units.

In conclusion, it can be said that the designed control system is well suited for its task. The response time is determined by the speed at which urea diffuses into the membrane and is not limited by the controller. In a forthcoming paper [5] more experimental results of this new sensor will be given.

\section{References}

1 B. H. van der Schoot and P. Bergveld, ISFET-based enzyme sensors, Biosensors, $3(1987 / 88) 161-186$.

2 B. H. van der Schoot and P. Bergueld, An ISFET-based microlitre titrator; integration of a chemical sensor-actuator system, Sensors and Actuators, 8 (1985) 11-22.

3 G. G. Guilbault, Analytical Uses of Immobilized Enzymes, Marcel Dekker, New York, 1984, Ch. 2.

4 B. H. van der Schoot, P. Bergveld and J. H. L. Onokiewicz, Development of a microprocessor-controlled system for stable pH control, Anal. Chim. Acta, 151 (1983) 143-151.

5 B. H. van der Schoot and P. Bergveld, The pH-static enzyme sensor; evaluation of the sensor properties, submitted for publication. 